\title{
Boundary regularity for the supercritical Lane-Emden heat flow
}

\author{
Simon Blatt ${ }^{1}$. Michael Struwe ${ }^{2}$
}

Received: 17 December 2014 / Accepted: 30 April 2015 / Published online: 7 June 2015

(C) Springer-Verlag Berlin Heidelberg 2015

\begin{abstract}
We establish a Pacard-type monotonicity formula and Morrey bounds up to the boundary for smooth solutions of the Lane-Emden heat flow $u_{t}-\Delta u=|u|^{p-2} u$ on a general, smoothly bounded domain $\Omega \subset \mathbb{R}^{n}, n \geq 3$, for exponents $p>2^{*}=2 n /(n-2)$, extending our previous work on the problem. As a consequence we obtain partially regular, self-similar tangent maps at any first blow-up point of the flow, and partial regularity at the blow-up time if the energy is uniformly bounded from below.
\end{abstract}

\section{Mathematics Subject Classification $\quad 35 \mathrm{~K} 58 \cdot 35 \mathrm{~K} 91$}

\section{Introduction}

Let $\Omega$ be a smoothly bounded domain in $\mathbb{R}^{n}, n \geq 3$. In [1] we derived a Pacard-type monotonicity formula for the flow

$$
u_{t}-\Delta u=|u|^{p-2} u \quad \text { on } \Omega \times[0, T[, \quad u=0 \quad \text { on } \partial \Omega \times[0, T[,
$$

for any $T>0$ in the "supercritical" case when $p>2^{*}=2 n /(n-2)$. For given initial data $u_{0}$ we showed that the solution $u$ to (1.1) up to a constant preserves the $L^{p, \mu}(\Omega)$-Morrey norm of the data, where $\mu=\frac{2 p}{p-2}<n$, and we derived various consequences from this fact. In the case of a convex domain our estimates extended up to the boundary and, in particular, we were able to show that the initial value problem for (1.1) is well-posed for arbitrary $T>0$

Communicated by L. Ambrosio.

S. B. gratefully acknowledges the support of the Forschungsinstitut für Mathematik (FIM), ETH Zurich.

\footnotetext{
Michael Struwe

michael.struwe@math.ethz.ch

Simon Blatt

simon.blatt@sbg.ac.at

1 Fachbereich Mathematik, Universität Salzburg, 5020 Salzburg, Austria

2 Departement Mathematik, ETH-Zürich, 8092 Zürich, Switzerland
} 
even for data $u_{0} \in H^{1} \cap L^{p, \mu}(\Omega)$ with $\nabla u_{0} \in L^{2, \mu}(\Omega)$, provided that $\left\|u_{0}\right\|_{L^{p, \mu}}+\left\|\nabla u_{0}\right\|_{L^{2, \mu}}$ is sufficiently small; see [1], Section 6.5.

Our aim in the present paper is to show that similar results also hold on a general domain. Moreover, for a smooth solution of (1.1) blowing up at time $T>0$ with uniformly bounded energy, we establish partial regularity at blow-up time in the sense that the set of singular points of the flow at time $T$ has codimension $\mu-1$, which in particular rules out blow-up away from the origin of any radially symmetric solution of (1.1) on a ball. Finally, if the domain is convex we also are able to show that the set of singular points of the flow on the boundary at time $T$ has codimension $\mu-1$ within $\partial \Omega$.

\section{Background}

\subsection{Notation}

Throughout the following a function $u$ will be called a smooth solution of (1.1) if $u \in$ $C^{1}(\bar{\Omega} \times[0, T[)$ solves $(1.1)$ in the sense of distributions. Standard regularity theory then shows that $u$ also is of class $C^{2}$ with respect to $x$ and satisfies (1.1) classically. Higher regularity (to the extent allowed by smoothness of the nonlinearity $g(v)=|v|^{p-2} v$ ) then follows from Schauder theory. The letter $C$ will designate a generic constant, sometimes numbered for clarity.

\subsection{Energy identity and consequences}

It is well-known that the flow (1.1) may be regarded as the $L^{2}$-gradient flow for the energy

$$
E(u)=\frac{1}{2} \int_{\Omega}|\nabla u|^{2} d x-\frac{1}{p} \int_{\Omega}|u|^{p} d x, \quad u \in H_{0}^{1} \cap L^{p}(\Omega) .
$$

In fact, multiplying (1.1) with $u_{t}$ and integrating by parts we obtain the energy identity

$$
E(u(t))+\int_{0}^{t} \int_{\Omega}\left|u_{t}\right|^{2} d x d s=E\left(u_{0}\right)
$$

for any smooth solution of (1.1) on $\Omega \times[0, T]$ with initial data $u(0)=u_{0}$. In particular, the energy function $t \mapsto E(u(t))$ is non-increasing. Moreover, whenever for some constant $E_{T}>-\infty$ and all $t \in\left[0, T\left[\right.\right.$ there holds $E(u(t)) \geq E_{T}$, we have $u_{t} \in L^{2}(\Omega \times[0, T[)$ with

$$
\int_{0}^{T} \int_{\Omega}\left|u_{t}\right|^{2} d x d s \leq E\left(u_{0}\right)-E_{T}
$$

and there exists a trace $u(T)=\lim _{t \uparrow T} u(t) \in L^{2}(\Omega)$ at the final time $T$. In addition, from Hölder's inequality we obtain the estimate

$$
\sup _{0 \leq t \leq T}\left|\|u(t)\|_{L^{2}}-\left\|u_{0}\right\|_{L^{2}}\right| \leq \int_{0}^{T}\left\|u_{t}(s)\right\|_{L^{2}} d s \leq \sqrt{T\left(E\left(u_{0}\right)-E_{T}\right)} .
$$

Moreover, multiplying (1.1) with $u$ and integrating we find

$$
\begin{aligned}
\frac{1}{2} \frac{d}{d t}\|u(t)\|_{L^{2}}^{2} & =\int_{\Omega \times\{t\}} u_{t} u d x=-\int_{\Omega \times\{t\}}\left(|\nabla u|^{2}-|u|^{p}\right) d x \\
& =-2 E(u(t))+\frac{p-2}{p}\|u(t)\|_{L^{p}}^{p}=-p E(u(t))+\frac{p-2}{2}\|\nabla u(t)\|_{L^{2}}^{2}
\end{aligned}
$$


for any $0<t<T$. Upon integrating this identity over the interval ]0, $T$ [, we then obtain the bound

$$
\begin{aligned}
& (p-2) \int_{0}^{T} \int_{\Omega}\left(\frac{|\nabla u|^{2}}{2}+\frac{|u|^{p}}{p}\right) d x d t \\
& =(p+2) \int_{0}^{T} E(u(t)) d t+\|u(T)\|_{L^{2}}^{2}-\left\|u_{0}\right\|_{L^{2}}^{2} \\
& \leq T(p+2) E\left(u_{0}\right)+\left\|u_{0}\right\|_{L^{2}}^{2}+2 T\left(E\left(u_{0}\right)-E_{T}\right) .
\end{aligned}
$$

for $u$ in $H^{1} \cap L^{p}\left(\Omega \times\left[0, T[)\right.\right.$ in terms of the data $u_{0} \in H^{1} \cap L^{p}(\Omega)$ and a lower bound for the energy.

Finally, as we had shown in [1, Lemma 6.3], given any smooth solution $u$ to (1.1), for any $0<t_{1}<T$ a bound similar to (2.5) on the interval [0, $t_{1}$ [ may be derived even without a uniform lower bound for the energy. Indeed, let $t_{0} \geq 0$ be minimal such that $E(u(t)) \leq 0$ for $t \geq t_{0}$. (If no such $t_{0}$ exists, (2.5) already yields the desired bound on [0,T [.) Letting

$$
g(t)=\int_{t_{0}}^{t}\|u(s)\|_{L^{p}}^{p} d s
$$

then with a uniform constant $c_{0}>0$ as in the proof of Lemma 6.3 in [1] we have

$$
\frac{d g}{d t}=\|u(t)\|_{L^{p}}^{p} \geq c_{0} g^{p / 2} \quad \text { for } t_{0} \leq t<T .
$$

Integration of this differential inequality from any $t_{1} \in\left[t_{0}, T\right.$ [ up to time $T$ gives the bound

$$
\int_{t_{0}}^{t_{1}} \int_{\Omega}|u|^{p} d x d t=g\left(t_{1}\right) \leq\left(c_{0} \frac{p-2}{2}\left(T-t_{1}\right)\right)^{-\frac{2}{p-2}} .
$$

Together with the energy inequality (2.1) we hence find the estimate

$$
\int_{t_{0}}^{t_{1}} \int_{\Omega}\left(|\nabla u|^{2}+|u|^{p}\right) d x d t \leq 2\left(t_{1}-t_{0}\right) E\left(u_{0}\right)+C\left(T-t_{1}\right)^{-\frac{2}{p-2}},
$$

which may be coupled with $(2.5)$ on $\left[0, t_{0}\right.$ [ to give the bound

$$
\int_{0}^{t_{1}} \int_{\Omega}\left(|\nabla u|^{2}+|u|^{p}\right) d x d t \leq C T E_{+}\left(u_{0}\right)+C\left\|u_{0}\right\|_{L^{2}}^{2}+C\left(T-t_{1}\right)^{-\frac{2}{p-2}}
$$

with a uniform constant $C>0$ for any $t_{1}<T$, where $E_{+}\left(u_{0}\right)=\max \left\{E\left(u_{0}\right), 0\right\}$.

\subsection{Morrey spaces}

Recall that for any $1 \leq p<\infty, 0<\lambda<n+2$ a function $f \in L^{p}(E)$ on $E \subset \mathbb{R}^{n} \times \mathbb{R}$ belongs to the parabolic Morrey space $L^{p, \lambda}(E)$ if

$$
\|f\|_{L^{p, \lambda}(E)}^{p}:=\sup _{z_{0}=\left(x_{0}, t_{0}\right) \in R^{n+1}, r>0} r^{\lambda-(n+2)} \int_{P_{r}\left(z_{0}\right) \cap E}|f|^{p} d z<\infty,
$$

where $P_{r}(x, t)$ denotes the backwards parabolic cylinder $\left.B_{r}(x) \times\right] t-r^{2}, t[$. In what follows the Morrey exponent $\lambda=\frac{2 p}{p-2}=\mu$, as defined above, will occur naturally. 


\subsection{The local monotonicity formula}

We recall some basic notation from [1]. Fix a point $x_{0} \in \Omega$ and a time $t_{0}>0$. Given a solution $u$ to (1.1) on $\Omega \times\left[0, t_{0}[\right.$, for $R>0$ let

$$
u_{R}(x, t)=R^{2 /(p-2)} u\left(x_{0}+R x, t_{0}+R^{2} t\right) \text { on } \Omega_{R} \times\left[-\frac{t_{0}}{R^{2}}, 0[,\right.
$$

where $\Omega_{R}=\left\{x ; x_{0}+R x \in \Omega\right\}$. Also let

$$
G(x, t)=\frac{1}{(4 \pi|t|)^{n / 2}} e^{-\frac{|x|^{2}}{4|t|}}, \quad x \in \mathbb{R}^{n}, t<0,
$$

be the fundamental solution to the heat equation with singularity at $(0,0)$, and set

$$
G_{\left(x_{0}, t_{0}\right)}(x, t)=G\left(x-x_{0}, t-t_{0}\right), \quad G_{*}(x)=G(x,-1)=(4 \pi)^{-n / 2} e^{-|x|^{2} / 4}
$$

for brevity. We sometimes also write $z=(x, t)$ for a generic point in space-time. Let $\varphi=\varphi(|x|) \in C^{\infty}\left(\mathbb{R}^{n}\right)$ be a compactly supported cut-off function such that $0 \leq \varphi \leq 1$, and for any $R>0$ set

$$
\varphi_{R}(x)=\varphi(R x) .
$$

Given $x_{0}, t_{0}, R>0$ let $u_{R}$ be as in (2.7) above and define

$$
\begin{aligned}
D^{\varphi}(R) & =\frac{1}{2} \int_{\Omega_{R}}\left|\nabla u_{R}(x,-1)\right|^{2} \varphi_{R}^{2}(x) G_{*}(x) d x \\
& =\frac{R^{\mu}}{2} \int_{\Omega \times\left\{t_{0}-R^{2}\right\}}|\nabla u|^{2} \varphi^{2}\left(x-x_{0}\right) G_{\left(x_{0}, t_{0}\right)} d x .
\end{aligned}
$$

For $q \geq 2$ we also let

$$
\begin{aligned}
F_{q}^{\varphi}(R) & =\frac{1}{q} \int_{\Omega_{R}}\left|u_{R}(x,-1)\right|^{q} \varphi_{R}^{2}(x) G_{*}(x) d x \\
& =\frac{R^{\frac{2 q}{p-2}}}{q} \int_{\Omega \times\left\{t_{0}-R^{2}\right\}}|u|^{q} \varphi^{2}\left(x-x_{0}\right) G_{\left(x_{0}, t_{0}\right)} d x .
\end{aligned}
$$

Then by [1], Proposition 3.1 for the function

$$
H^{\varphi}(R)=H_{\left(x_{0}, t_{0}\right)}^{\varphi}(R):=\frac{p-2}{p} F_{p}^{\varphi}(R)+\frac{1}{2 p}\left(R \frac{d}{d R} F_{2}^{\varphi}(R)-A_{2}^{\varphi}(R)\right)
$$

with

$$
\begin{aligned}
A_{2}^{\varphi}(R)= & \frac{R^{\mu-2}}{2} \int_{\Omega \times\left\{t_{0}-R^{2}\right\}}|u|^{2} x \cdot \nabla \varphi^{2} G_{\left(x_{0}, t_{0}\right)} d x \\
& +2 R^{\mu} \int_{\Omega \times\left\{t_{0}-R^{2}\right\}} u \nabla u \cdot \nabla \varphi^{2} G_{\left(x_{0}, t_{0}\right)} d x
\end{aligned}
$$

there holds the following perturbed monotonicity formula.

Proposition 2.1 ([1], Propositions 3.1 and 3.18) Let $u$ be a smooth solution of (1.1). Then after translating $x_{0}=0$, there holds

$$
\begin{aligned}
R \frac{d}{d R} H^{\varphi}(R) \geq & \frac{R^{\mu}}{2 p} \int_{\Omega \times\left\{t_{0}-R^{2}\right\}} \frac{\left|x \cdot \nabla u+2\left(t-t_{0}\right) u_{t}+a u\right|^{2}}{\left|t_{0}-t\right|} \varphi^{2} G_{\left(x_{0}, t_{0}\right)} d x \\
& +\frac{2}{p}\left(A_{0}^{\varphi}+B^{\varphi}\right),
\end{aligned}
$$


where $a=\frac{2}{p-2}$ and where $A_{0}^{\varphi}$ denotes the error term

$$
\begin{aligned}
A_{0}^{\varphi}(R)= & R^{\mu} \int_{\Omega \times\left\{t_{0}-R^{2}\right\}}\left(|\nabla u|^{2}-\frac{2}{p}|u|^{p}+R^{-2} \frac{|u|^{2}}{p-2}\right) \varphi x \cdot \nabla \varphi G_{\left(x_{0}, t_{0}\right)} d x \\
& -4 R^{\mu+2} \int_{\Omega \times\left\{t_{0}-R^{2}\right\}}|\nabla u|^{2}|\nabla \varphi|^{2} G_{\left(x_{0}, t_{0}\right)} d x .
\end{aligned}
$$

Finally, $B^{\varphi}=B^{\varphi}(R)$ is the boundary term

$$
B^{\varphi}(R)=\frac{R^{\mu}}{2} \int_{\partial \Omega \times\left\{t_{0}-R^{2}\right\}} v \cdot x|\nabla u|^{2} \varphi^{2} G_{\left(x_{0}, t_{0}\right)} d o,
$$

where $v$ is the outward unit normal.

Moreover, also using the Giga-Kohn [7] expression

$$
H^{\varphi}(R)=\frac{2}{p} D^{\varphi}(R)-\frac{2}{p} F_{p}^{\varphi}(R)+\frac{2}{p(p-2)} F_{2}^{\varphi}(R)
$$

for $H^{\varphi}$, we are able to express

$$
\begin{aligned}
H^{\varphi}(R)= & \frac{2(p-2)}{p(p+2)}\left(D^{\varphi}(R)+F_{p}^{\varphi}(R)\right) \\
& +\frac{2}{p(p+2)}\left(\frac{d}{d R}\left(R F_{2}^{\varphi}(R)\right)-A_{2}^{\varphi}(R)\right) .
\end{aligned}
$$

Remark 2.2 With the expression (2.12) for $H$ and with weight $\varphi \equiv 1$, that is, without localization, monotonicity of $H$ was observed by Giga-Kohn [7] in the form of an energy inequality for the function $w(y, s)=\left(t_{0}-t\right)^{1 /(p-2)} u(x, t)$ obtained from writing $u$ in selfsimilar variables $y=x / \sqrt{t_{0}-t}, s=\log \left(1 /\left(t_{0}-t\right)\right)$. On the other hand, motivated by monotonicity formulas for harmonic maps, in [13] the heat kernel $G$ was introduced as a weight and a natural monotonicity formula was derived for maps between manifolds evolving under the heat flow for the Dirichlet energy. When applied to Eq. (1.1), these techniques yield the Giga-Kohn [7] monotonicity result without their assumption on the type of blow-up and on any convex domain, and allow further conclusions. For instance, in [3] the techniques of [13] were applied to prove partial regularity of borderline solutions of (1.1)

The key novel feature of Proposition 2.1 is that the difference of the two leading terms in (2.12) when passing to the equivalent expression (2.13) magically is converted into their sum (up to an error term of lower order). This possible conversion was first observed by Pacard [12] in the time-independent case of (1.1) through clever manipulations of the standard approach. In our paper [1] we showed that the techniques from [13] may be used to carry over Pacard's idea to the time-dependent setting.

\section{Results}

Fix a smooth cut-off function $\eta$ such that $\chi_{B_{1}(0)} \leq \eta \leq \chi_{B_{2}(0)},|\nabla \eta| \leq 4$. For any $0<\rho \leq \infty$ then let $\varphi(x)=\varphi^{\rho}(x)=\eta(x / \rho)$ and set

$$
\delta(\rho, R)= \begin{cases}R^{-n} e^{-\frac{\rho^{2}}{4 R^{2}}}, & \text { if } R \leq \rho, \\ \rho^{-n}\left(1+R^{2} \rho^{\mu-2}\right), & \text { else. }\end{cases}
$$


Our main goal in this note is to obtain the following result which extends our previous result [1], Proposition 6.1, to the boundary.

In the following, for given $u_{0}$ and $T>0$ we denote as $C_{0}$ a constant depending only on $E\left(u_{0}\right),\left\|u_{0}\right\|_{L^{2}}$, and $T$.

Proposition 3.1 There are constants $C=C(\Omega), R_{0}=R_{0}(\Omega)>0$ such that for any smooth solution $u$ of (1.1) on $\Omega \times\left[0, T\left[\right.\right.$ with initial data $u(0)=u_{0}$, any $\rho>0$, any $x_{1} \in \bar{\Omega}$, any $0<t_{1}<T$, and any $0<r<R \leq \min \left\{R_{0}, \sqrt{2} \rho, \sqrt{t_{1}}\right\}$ with $\varphi=\varphi^{\rho}$ there holds

$$
H_{\left(x_{1}, t_{1}\right)}^{\varphi}(r) \leq H_{\left(x_{1}, t_{1}\right)}^{\varphi}(R)+C R \sup _{x_{0} \in \Omega} H_{\left(x_{0}, t_{1}\right)}^{\varphi}(R)+C_{0} \delta(\rho, R) .
$$

The constant $R_{0}>0$ is determined in Lemma 4.3 where we also assume that $R_{0} \leq \sqrt{2} r_{0}$ with $r_{0}>0$ as in (4.1).

Just as in [1], Proposition 6.2, the previous proposition implies the following Morrey bound.

Proposition 3.2 There are constants $C=C(\Omega), R_{0}=R_{0}(\Omega)>0$ such that for any smooth solution $u$ of (1.1) on $\Omega \times\left[0, T\right.$, any $\rho>0$, any $x_{0} \in \bar{\Omega}$, and any $0<2 r<R_{1} \leq$ $\min \left\{R_{0}, \sqrt{2} \rho, \sqrt{T / 2}\right\}$ letting $t_{0}=T-r^{2}$ with $\varphi=\varphi^{\rho}$ there holds

$$
\begin{aligned}
& \|\nabla u\|_{L^{2, \mu}\left(Q_{r}\left(x_{0}, t_{0}\right)\right)}^{2}+\|u\|_{L^{p, \mu}\left(Q_{r}\left(x_{0}, t_{0}\right)\right)}^{p} \\
& \leq C \sup _{x_{1} \in \Omega} H_{\left(x_{1}, T\right)}^{\varphi}\left(R_{1}\right)+C R_{1} \sup _{x_{1} \in \Omega} H_{\left(x_{1}, T\right)}^{\varphi}\left(R_{1}\right)+C_{0} \delta\left(\rho, R_{1}\right),
\end{aligned}
$$

where $Q_{r}\left(x_{0}, t_{0}\right)=P_{r}\left(x_{0}, t_{0}\right) \cap \Omega \times[0, T[$.

Remark 3.3 As in [1], Section 6.5, from Proposition 3.2 it follows that the initial value problem for (1.1) is well-posed for initial data $u_{0} \in H^{1} \cap L^{p, \mu}(\Omega)$ with $\nabla u_{0} \in L^{2, \mu}(\Omega)$ satisfying

$$
\sup _{x_{0} \in R^{n}, 0<r<r_{0}} r^{\mu-n} \int_{\Omega_{r}\left(x_{0}\right)}\left(\left|\nabla u_{0}\right|^{2}+\left|u_{0}\right|^{p}\right) d x<\varepsilon
$$

for sufficiently small $\varepsilon>0$ and some number $r_{0}=r_{0}(\varepsilon)>0$. Here, $\Omega_{r}\left(x_{0}\right)=B_{r}\left(x_{0}\right) \cap \Omega$. Indeed, letting $u_{k}$ be the solutions to the Cauchy problem for (1.1) for suitable smooth data $u_{0 k}$ such that $u_{0 k} \rightarrow u_{0}$ in $H^{1} \cap L^{p}(\Omega)$ as $k \rightarrow \infty$, from Proposition 3.2 and the $\varepsilon$-regularity result [1], Proposition 4.1, we find the a-priori bound

$$
\left\|u_{k}(t)\right\|_{L^{\infty}(\Omega)} \leq C t^{-\frac{1}{p-2}}
$$

on a uniform time interval $0<t<T$, and $\left(u_{k}\right)$ converges to a smooth solution $u$ of (1.1) with data $u_{0}$. Moreover, given $\delta>0$, after replacing $\varepsilon$ with a smaller number $\varepsilon>0$, if necessary, we can achieve that there holds $\|u(T)\|_{L^{\infty}(\Omega)} \leq \delta$.

Provided also $\delta>0$ is sufficiently small, by standard results as given, for instance, in [14], Theorem 3(b), and [8], the solution $u$ then may be continued for all time and $u(t) \rightarrow 0$ as $t \rightarrow \infty$.

Propositions 3.1 and 3.2 together with the $\varepsilon$-regularity result [1], Proposition 4.1, give rise to the following global analogue of [1], Proposition 6.8 .

Proposition 3.4 Let $\Omega \subset \mathbb{R}^{n}$ be smooth and bounded, and let $\rho>0$. Given smooth data $u_{0}$, let $u \in C^{1}(\bar{\Omega} \times[0, T[)$ be the unique maximal smooth solution to (1.1) with initial data $u(0)=u_{0}$. Then for any $x_{0} \in \bar{\Omega}$, either 
(i) there is $r>0$ such that $u$ extends smoothly to the closure of $Q_{r}\left(x_{0}, T\right)$, or

(ii) there exists $R_{1}>0$ independent of $x_{0}$ such that for any $0<R<R_{1}$ there holds $H_{\left(x_{0}, T\right)}^{\varphi}(R) \geq \varepsilon_{0}$, where $\varphi=\varphi^{\rho}$ and where $\varepsilon_{0}>0$ is a constant independent of $x_{0}$ and $u$.

Finally, we also have the following result, extending the results of [3] to the case of a finite time interval.

Proposition 3.5 Consider a family of smooth solution $u_{k}$ of (1.1) on $\Omega \times[0, T[, T<\infty$, $k \in \mathbb{N}$, and assume that $u_{k} \stackrel{w}{\rightarrow} u$ weakly in $H^{1} \cap L^{p}(\Omega \times[0, T[)$ as $k \rightarrow \infty$. Then $u$ weakly solves (1.1) on $\Omega \times[0, T[$ and for any compact set $Q \subset \bar{\Omega} \times] 0, T]$ there holds $\mathcal{H}^{n+2-\mu}(\operatorname{sing}(u) \cap Q)<\infty$, where $\operatorname{sing}(u)$ is the set of points $\left.\left.\left(x_{0}, t_{0}\right) \in \bar{\Omega} \times\right] 0, T\right]$ such that $u$ cannot be extended smoothly to a neighborhood of $\left(x_{0}, t_{0}\right)$ in $\left.\left.\bar{\Omega} \times\right] 0, T\right]$.

Remark 3.6 By (2.2) and (2.5) any sequence $\left(u_{k}\right)$ of smooth solutions of (1.1) with the property that with constants $-\infty<E_{t_{1}} \leq E_{0}<\infty, C>0$ for any $0 \leq t_{1}<T$ there holds

$$
\left\|u_{k}(0)\right\|_{L^{2}(\Omega)} \leq C, E_{t_{1}} \leq E\left(u_{k}(t)\right) \leq E_{0} \quad \text { for all } 0 \leq t \leq t_{1}, k \in \mathbb{N},
$$

is bounded locally in $H^{1} \cap L^{p}(\bar{\Omega} \times[0, T[)$ and hence has a locally weakly convergent subsequence. In fact, by (2.6) and a standard measure theoretic argument (3.2) holds for any sequence $\left(u_{k}\right)$ of smooth solutions of (1.1) on $\left[0, T\left[\right.\right.$ such that $\left\|u_{k}(0)\right\|_{L^{2}(\Omega)}+E\left(u_{k}(0)\right) \leq C$, uniformly in $k \in \mathbb{N}$.

As a consequence of Propositions 3.4 and 3.5 we obtain the existence of a non-trivial self-similar tangent map at any first blow-up point $\left(x_{0}, T\right)$, analogous to Theorem 6.9 in [1], but now allowing also $x_{0} \in \partial \Omega$.

Theorem 3.7 Let $\Omega \subset \mathbb{R}^{n}$ be smooth and bounded. For given smooth data $u_{0}$ let $u \in$ $C^{1}\left(\bar{\Omega} \times\left[0, T[)\right.\right.$ be the unique maximal smooth solution to (1.1) with initial data $u_{0}$, and let $\left(x_{0}, T\right) \in \operatorname{sing}(u) \cap \bar{\Omega} \times\{T\}$ be a first blow-up point as defined in Proposition 3.5. Shift coordinates so that $\left(x_{0}, T\right)=(0,0)$. Then the following holds:

(i) There exists a sequence $R_{k} \rightarrow 0$ and a self-similar "ancient" weak solution $\bar{u} \neq 0$ on $\left.\mathbb{R}^{n} \times\right]-\infty, 0\left[\right.$ or on the half-space $\left.\mathbb{R}_{+}^{n} \times\right]-\infty, 0[$ which is smooth away from a set $\sin g(\bar{u})$ of locally finite $(n+2-\mu)$-dimensional Hausdorff measure such that $u_{k}=u_{R_{k}} \rightarrow \bar{u}$ as $k \rightarrow \infty$, smoothly locally away from sing $(\bar{u})$ and from $t=0$.

Here for any $R>0$ we let

$$
u_{R}(x, t)=R^{2 /(p-2)} u\left(R x, R^{2} t\right)
$$

moreover, by definition $\bar{u}$ is self-similar if $\bar{u} \equiv \bar{u}_{R}$ for all $R>0$.

(ii) If $\left(x_{0}, T\right)=(0,0)$ is of Type I in the sense that there exist constants $C, r_{0}>0$ such that

$$
\limsup _{t \uparrow 0}\left(|t|^{1 /(p-2)} \sup _{x \in \Omega_{r_{0}}(0)}|u(x, t)|\right) \leq C .
$$

then $\bar{u}$ is smooth and $\left(u_{k}\right)$ converges smoothly to $\bar{u}$, locally away from $t=0$.

(iii) If $\left(x_{0}, T\right)=(0,0)$ is not of Type $I$, in addition to the above there exist sequences $R_{k} \rightarrow 0, t_{k} \uparrow 0, \Omega \ni x_{k} \rightarrow 0$, and an "eternal" solution $\bar{u} \neq 0$ of (1.1) on $\mathbb{R}^{n} \times \mathbb{R}$ or on the half-space $\mathbb{R}_{+}^{n} \times \mathbb{R}$ with

$$
\|\bar{u}\|_{L^{\infty}}=|\bar{u}(0,0)|=1
$$


and such that

$$
u_{k}(x, t)=R_{k}^{\frac{2}{p-2}} u\left(x_{k}+R_{k} x, t_{k}+R_{k}^{2} t\right) \rightarrow \bar{u}
$$

smoothly locally as $k \rightarrow \infty$.

The proof of Theorem 3.7 is almost identical with the proof of Theorem 6.9 in [1]. For completeness, we indicate the few necessary modifications in the next section.

Under suitable additional assumptions we also can characterize the behavior of a solution $u$ to (1.1) blowing up at time $T<\infty$ up to its maximal time of existence. A natural condition is to require the existence of

$$
\liminf _{t \uparrow T} E(u(t))=E_{T}>-\infty,
$$

ensuring $L^{2}$-convergence $u(t) \rightarrow u(T)$ as $t \uparrow T$, as we had seen in Sect. 2.2. Conversely, it seems very likely that failure of (3.3) will be associated with complete blow-up of $u$. See Remark 3.9 below for some results in this regard and further references.

Ideally, we would wish to show that whenever (3.3) holds we have $u_{1}:=u(T) \in H^{1} \cap$ $L^{p}(\Omega)$ with $u_{1} \in L^{p, \mu}, \nabla u_{1} \in L^{2, \mu}$, and that we can restart the flow with $u_{1}$ as initial data. In order to improve our estimates for $u$ at time $T$ we note that, similar to (2.5), from (2.4) by Hölder's inequality for any $t<T$ we have

$$
\int_{\Omega \times\{t\}}\left(|\nabla u|^{2}+|u|^{p}\right) d x \leq 2 \mu E(u(t))+C\|u(t)\|_{L^{2}(\Omega)}\left\|u_{t}(t)\right\|_{L^{2}(\Omega)} .
$$

Now (2.3) and (3.3) give a uniform bound $\sup _{0 \leq t<T}\|u(t)\|_{L^{2}(\Omega)} \leq C_{T}$, where we denote as $C_{T}$ any constant depending only on $E\left(u_{0}\right),\left\|u_{0}\right\|_{L^{2}}, T$, and $E_{T}$. Thus, given $0<R<\sqrt{T}$, upon integrating (3.4) over [ $T-R^{2}, T$ [ and using (2.1) and again Hölder's inequality we obtain

$$
\int_{T-R^{2}}^{T} \int_{\Omega}\left(|\nabla u|^{2}+|u|^{p}\right) d x d t \leq C R^{2} E_{+}\left(u_{0}\right)+C_{T} R\left\|u_{t}\right\|_{L^{2}(\Omega \times[0, T[)} \leq C_{T} R .
$$

More generally, we may require that for some constant $\kappa>0$ there holds

$$
\liminf _{R \downarrow 0} R^{-\kappa} \int_{T-R^{2}}^{T} \int_{\Omega}\left(|\nabla u|^{2}+|u|^{p}\right) d x d t<\infty .
$$

Observe that (3.6) with $\kappa=2$ together with (2.1) implies (3.3) and weak convergence $u(t) \rightarrow u(T)$ in $H^{1} \cap L^{p}(\Omega)$. Conversely, (3.3) yields (3.6) with $\kappa=1$. Thus, the interval $1 \leq \kappa \leq 2$ is a natural range for $\kappa$.

Theorem 3.8 Let $u$ be a smooth solution of (1.1) on $\Omega \times[0, T[$ for some $T<\infty$, and assume that (3.6) holds for some $1 \leq \kappa \leq 2$. Then $\mathcal{H}^{n+2-\kappa-\mu}(\operatorname{sing}(u) \cap \bar{\Omega} \times\{T\})<\infty$, where sing $(u)$ is as in Proposition 3.5 above. In particular, if (3.3) holds we have $\mathcal{H}^{n+1-\mu}(\operatorname{sing}(u) \cap \bar{\Omega} \times\{T\})<\infty$.

Remark 3.9 In the special case when $\Omega$ is a ball and when $u$ is a radially symmetric smooth solution of (1.1) on $\Omega \times[0, T$ [ blowing up at time $T>0$, by Theorem 3.8 either $E(u(t)) \rightarrow$ $-\infty$ as $t \uparrow T$, or $u$ can only blow up at the origin, which rules out one of the possible blow-up scenarios described in [9], Theorem 1.11, or [10], Theorem 1.2. Indeed, if (3.3) holds, by Theorem 3.8 we have that $\mathcal{H}^{n+1-\mu}(\operatorname{sing}(u) \cap \Omega \times\{T\})=0$. Recalling that $\mu>2$, then we see that $\operatorname{sing}(u) \cap \Omega \times\{T\}$ cannot contain a sphere. 
On a convex domain, together with an observation by De Marchis et al. [4] our estimates also give boundary regularity for solutions $u>0$ of (1.1) up to a set of the same codimension as in Theorem 3.8 for interior regularity.

Theorem 3.10 Suppose that $\Omega$ is convex and that $u$ is a smooth solution of (1.1) on $\Omega \times[0, T[$ for some $T<\infty$ with initial data $0 \leq u_{0} \in C^{1}(\bar{\Omega})$ and assume that (3.6) holds for some $1 \leq \kappa \leq 2$. Then we have $\mathcal{H}^{n+1-\kappa-\mu}(\operatorname{sing}(u) \cap \partial \Omega \times\{T\})<\infty$. In particular, if (3.3) holds, we have $\mathcal{H}^{n-\mu}(\operatorname{sing}(u) \cap \partial \Omega \times\{T\})<\infty$; moreover, if we assume that (3.6) holds for some $\kappa>n+1-\mu$, the function $u$ extends smoothly to a neighborhood of $\partial \Omega \times\{T\}$.

Remark 3.11 Note that for $2^{*}<p<2^{+}=\frac{2(n-1)}{n-3}$ we have $n-1<\mu<n$; hence if (3.6) holds for $\kappa=2$ and if $\Omega$ is convex, we have full boundary regularity in this case.

\section{Proofs of Propositions 3.1-3.5 and of Theorems 3.7 and 3.8}

For any smooth, bounded $\Omega \subset \mathbb{R}^{n}$ we can find a number $C_{\Omega} \geq 0$ such that for any choice of origin $x_{1}=0 \in \Omega$ with $r_{1}=\operatorname{dist}\left(x_{1}, \partial \Omega\right)>0$ there holds

$$
x \cdot v(x) \geq r_{1}-C_{\Omega}|x|^{2} \text { for all } x \in \partial \Omega .
$$

Indeed, choosing coordinates $x=\left(x^{\prime}, x_{n}\right)$ for $\mathbb{R}^{n}$ so that $x_{0}:=\left(0, r_{1}\right) \in \partial \Omega$ there are neighborhoods $W$ of $x_{0}$ in $\mathbb{R}^{n}, U=B_{s}\left(0 ; \mathbb{R}^{n-1}\right)$ and a smooth map $h: U \rightarrow \mathbb{R}$ with $h(0)=r_{1}, d h(0)=0$ such that

$$
\partial \Omega \cap W=\mathcal{G}(h)=\left\{\left(x^{\prime}, h\left(x^{\prime}\right)\right) ; x^{\prime} \in U\right\}
$$

and

$$
v(x)=\left(-\nabla h\left(x^{\prime}\right), 1\right) / \sqrt{1+\left|\nabla h\left(x^{\prime}\right)\right|^{2}}=\left(O\left(\left|x^{\prime}\right|\right), 1+O\left(\left|x^{\prime}\right|^{2}\right)\right.
$$

for $x=\left(x^{\prime}, h\left(x^{\prime}\right)\right) \in \partial \Omega \cap W$; hence $x \cdot v(x)=r_{1}+O\left(\left|x^{\prime}\right|^{2}\right) \geq r_{1}-C|x|^{2}$ with a constant $C>0$ that may be chosen uniformly with respect to $x_{1} \in \Omega$.

Moreover, there is $r_{\Omega}>0$ such that for any $x_{0} \in \partial \Omega$ there is a ball $B_{r_{\Omega}}(x) \subset \Omega$ touching $\partial \Omega$ at $x_{0}$. We then set $r_{0}=\min \left\{r_{\Omega}, 1 /\left(8 C_{\Omega}\right)\right\}$, if $C_{\Omega}>0$, and otherwise let $r_{0}=r_{\Omega}$.

Note that for a convex domain $\Omega$ and any $x \in \partial \Omega$ we have

$$
r_{1} v(x) \in \overline{B_{r_{1}}(0)} \subset \bar{\Omega} \subset\left\{y \in \mathbb{R}^{n} ; y \cdot v(x) \leq x \cdot v(x)\right\},
$$

where $0=x_{1} \in \Omega, r_{1}=\operatorname{dist}\left(x_{1}, \partial \Omega\right)>0$ as above. Hence we may take $C_{\Omega}=0$ in this case.

Given any number $\rho>0$, let $\varphi=\varphi^{\rho}$ as above, and let $u$ be a smooth solution of (1.1) on $\Omega \times\left[0, T\right.$. For any choice of origin $x_{1}=0 \in \Omega$ and any $0<t_{1}<T$, with $\left(x_{1}, t_{1}\right)$ as center of scaling we have $r F_{2}^{\varphi}(r) \rightarrow 0$ as $r \downarrow 0$. Thus, for any $0<R \leq R_{1} \leq \sqrt{t_{1}}$ by (2.13) we obtain

$$
\frac{1}{R} \int_{0}^{R}\left(D^{\varphi}(r)+F_{p}^{\varphi}(r)\right) d r \leq \frac{C}{R} \int_{0}^{R} H^{\varphi}(r) d r+\frac{C}{R} \int_{0}^{R}\left|A_{2}^{\varphi}(r)\right| d r .
$$

On the other hand, from Proposition 2.1 for $0<r<R \leq R_{1}$ we obtain

$$
H^{\varphi}(r)+\frac{2}{p} \int_{r}^{R} B^{\varphi}(s) \frac{d s}{s} \leq H^{\varphi}(R)+\frac{2}{p} \int_{r}^{R}\left|A_{0}^{\varphi}(s)\right| \frac{d s}{s} .
$$


Letting $B_{ \pm}^{\varphi}$ denote the contributions to the boundary term $B^{\varphi}$ from points $x \in \partial \Omega$ where $\pm x \cdot v(x) \geq 0$, we thus have

$$
H^{\varphi}(r)+\frac{2}{p} \int_{r}^{R} B_{+}^{\varphi}(s) \frac{d s}{s} \leq H^{\varphi}(R)+\frac{2}{p} \int_{r}^{R}\left(\left|A_{0}^{\varphi}(s)\right|+\left|B_{-}^{\varphi}(s)\right|\right) \frac{d s}{s} .
$$

Together with (4.2) the bound (4.4) yields the inequality

$$
\begin{aligned}
& \frac{1}{R} \int_{0}^{R}\left(D^{\varphi}(r)+F_{p}^{\varphi}(r)\right) d r \\
& \quad \leq C H^{\varphi}(R)+\frac{C}{R} \int_{0}^{R}\left(\left|A_{2}^{\varphi}(r)\right|+\int_{r}^{R}\left(\left|A_{0}^{\varphi}(s)\right|+\left|B_{-}^{\varphi}(s)\right|\right) \frac{d s}{s}\right) d r .
\end{aligned}
$$

After using (4.4) again, from (4.4) for any $0<R \leq R_{1} \leq \sqrt{t_{1}}$ we find

$$
\begin{aligned}
& \frac{1}{R} \int_{0}^{R}\left(D^{\varphi}(r)+F_{p}^{\varphi}(r)\right) d r \\
& \quad \leq C H^{\varphi}\left(R_{1}\right)+\frac{C}{R} \int_{0}^{R}\left|A_{2}^{\varphi}(r)\right| d r+C \int_{0}^{R_{1}}\left(\left|A_{0}^{\varphi}(r)\right|+\left|B_{-}^{\varphi}(r)\right|\right) \frac{d r}{r} .
\end{aligned}
$$

With the help of [1], Lemma 6.3, or (2.6) above, the bound

$$
\frac{1}{R} \int_{0}^{R}\left|A_{2}^{\varphi}(r)\right| d r+\int_{0}^{R_{1}}\left|A_{0}^{\varphi}(r)\right| \frac{d r}{r} \leq C_{0} \delta\left(\rho, R_{1}\right)
$$

may be shown exactly as in [1], Lemma 6.4, where $C_{0}>0$ again is a constant depending only on $E\left(u_{0}\right),\left\|u_{0}\right\|_{L^{2}}$, and $T$. From (4.5) for any $0<R \leq R_{1} \leq \sqrt{t_{1}}$ we then obtain the bound

$$
\frac{1}{R} \int_{0}^{R}\left(D^{\varphi}(r)+F_{p}^{\varphi}(r)\right) d r \leq C H^{\varphi}\left(R_{1}\right)+C \int_{0}^{R_{1}}\left|B_{-}^{\varphi}(r)\right| \frac{d r}{r}+C_{0} \delta\left(\rho, R_{1}\right) .
$$

For $0<r \leq \sqrt{t_{1} / 2}$ define

$$
\beta_{t_{1}}(r):=r^{\mu-(n+1)} \sup _{x_{0} \in \Omega} \int_{\partial Q_{r}\left(x_{0}, t_{1}-r^{2}\right)}|\nabla u|^{2} d o d t
$$

where $\partial Q_{r}(x, t)=P_{r}(x, t) \cap\left(\partial \Omega \times\left[0, T[)\right.\right.$. Given $x_{1} \in \Omega$, for any $r>0$ we also set

$$
\partial \Omega_{r}=\partial \Omega_{r}\left(x_{1}\right)=B_{r}\left(x_{1}\right) \cap \partial \Omega, \partial \mathcal{A}_{r}=\partial \mathcal{A}_{r}\left(x_{1}\right)=\partial \Omega_{2 r} \backslash \partial \Omega_{r}
$$

for brevity.

Lemma 4.1 There is a constant $C=C(\Omega)$ such that for any $R_{1} \leq \sqrt{t_{1}}$ there holds

$$
\int_{0}^{R_{1}}\left|B_{-}^{\varphi}(r)\right| \frac{d r}{r} \leq C R_{1} \sup _{0<r<R_{1} / \sqrt{2}} \beta_{t_{1}}(r) .
$$

Proof Letting $R_{k}=2^{\frac{1-k}{2}} R_{1}, k \in \mathbb{N}$, decompose

$$
\int_{0}^{R_{1}}\left|B_{-}^{\varphi}(r)\right| \frac{d r}{r}=\sum_{k=1}^{\infty} \int_{R_{k+1}}^{R_{k}}\left|B_{-}^{\varphi}(r)\right| \frac{d r}{r} .
$$


For each $k \geq 2$ estimate (4.1) gives

$$
\begin{aligned}
\int_{R_{k}}^{R_{k-1}}\left|B_{-}^{\varphi}(r)\right| \frac{d r}{r} \leq & C \int_{R_{k}}^{R_{k-1}} r^{\mu-n-1} \int_{\partial \Omega \times\left\{t_{1}-r^{2}\right\}}|x|^{2}|\nabla u|^{2} \varphi^{2} e^{-\frac{|x|^{2}}{4 r^{2}}} d o d r \\
\leq & C \int_{R_{k}}^{R_{k-1}} r^{\mu-n-1} \int_{\partial \Omega_{R_{k}} \times\left\{t_{1}-r^{2}\right\}}|x|^{2}|\nabla u|^{2} e^{-\frac{|x|^{2}}{4 r^{2}}} d o d r \\
& +C \sum_{m \in \mathbb{N}_{0}} \int_{R_{k}}^{R_{k-1}} r^{\mu-n-1} \int_{\partial \mathcal{A}_{2^{m}} R_{k} \times\left\{t_{1}-r^{2}\right\}}|x|^{2}|\nabla u|^{2} e^{-\frac{|x|^{2}}{4 r^{2}}} d o d r \\
\leq & C R_{k}^{\mu-n} \int_{\partial Q_{R_{k}\left(x_{1}, t_{1}-R_{k}^{2}\right)}|\nabla u|^{2} d o d t} d t \\
& +C \sum_{m \in \mathbb{N}_{0}} R_{k}^{\mu-n} 2^{2 m} e^{-2^{2 m-3}} \int_{t_{1}-2 R_{k}^{2}}^{t_{1}-R_{k}^{2}} \int_{\partial \mathcal{A}_{2^{m} R_{k}} \times\{t\}}|\nabla u|^{2} d o d t .
\end{aligned}
$$

Since we can cover $\partial \mathcal{A}_{2^{m}} R_{k}$ by a set of at most $C 2^{m(n-1)}$ balls of radius $R_{k}$ centered on $\partial \Omega$ we can estimate this further to obtain

$$
\int_{R_{k}}^{R_{k-1}}\left|B_{-}^{\varphi}(r)\right| \frac{d r}{r} \leq C R_{k} \sum_{m \in \mathbb{N}_{0}} 2^{m(1+n)} e^{-2^{2 m-3}} \beta_{t_{1}}\left(R_{k}\right) \leq C R_{k} \cdot \beta_{t_{1}}\left(R_{k}\right)
$$

for each $k \geq 2$. With (4.8) this gives

$$
\int_{0}^{R_{1}}\left|B_{-}^{\varphi}(r)\right| \frac{d r}{r} \leq C R_{1} \sum_{k=2}^{\infty} 2^{-k / 2} \beta_{t_{1}}\left(R_{k}\right) \leq C R_{1} \sup _{0<r<R_{1} / \sqrt{2}} \beta_{t_{1}}(r),
$$

as claimed.

Conversely, we have the following result.

Lemma 4.2 With a constant $C=C(\Omega)$ for any $R_{1} \leq \min \left\{\sqrt{2} r_{0}, \sqrt{2} \rho, \sqrt{t_{1}}\right\}$ we have

$$
\sup _{0<r<R_{1} / \sqrt{2}} \beta_{t_{1}}(r) \leq C \sup _{x_{0} \in \Omega} \int_{0}^{R_{1}} B_{\left(x_{0}, t_{1}\right)}^{\varphi}(r) \frac{d r}{r}+C R_{1} \sup _{0<r<R_{1} / \sqrt{2}} \beta_{t_{1}}(r) .
$$

Proof For any $x_{0} \in \Omega$ let $B_{ \pm}^{\varphi}$ denote the contributions to the boundary term $B_{\left(x_{0}, t_{1}\right)}^{\varphi}$ from points $x \in \partial \Omega$ where $\pm x \cdot v(x) \geq 0$. Then by (4.1) for any number $0<R \stackrel{R}{<}$ $\min \left\{r_{0}, \rho, \sqrt{t_{1} / 2}\right\}$ we have

$$
\beta_{t_{1}}(R) \leq C \sup _{x_{0} \in \Omega, \operatorname{dist}\left(x_{0}, \partial \Omega\right)=R} \int_{R}^{\sqrt{2} R} B_{+}^{\varphi}(r) \frac{d r}{r} .
$$

Hence from Lemma 4.1 for any $R_{1} \leq \min \left\{\sqrt{2} r_{0}, \sqrt{2} \rho, \sqrt{t_{1}}\right\}$ it follows that

$$
\begin{aligned}
\sup _{0<r<R_{1} / \sqrt{2}} \beta_{t_{1}}(r) & \leq C \sup _{x_{0} \in \Omega} \int_{0}^{R_{1}} B_{+}^{\varphi}(r) \frac{d r}{r} \\
& \leq C \sup _{x_{0} \in \Omega} \int_{0}^{R_{1}} B_{\left(x_{0}, t_{1}\right)}^{\varphi}(r) \frac{d r}{r}+C R_{1} \sup _{0<r<R_{1} / \sqrt{2}} \beta_{t_{1}}(r),
\end{aligned}
$$

as desired.

Together with (4.4) the previous result gives the following estimate. 
Lemma 4.3 There are constants $C=C(\Omega), R_{0}=R_{0}(\Omega)>0$, such that for any $0<R<$ $\min \left\{R_{0}, \sqrt{2} \rho, \sqrt{t_{1}}\right\}$ there holds

$$
\sup _{0<r<R / \sqrt{2}} \beta_{t_{1}}(r) \leq C \sup _{x_{0} \in \Omega} H_{\left(x_{0}, t_{1}\right)}^{\varphi}(R)+C_{0} \delta(\rho, R) .
$$

Proof For sufficiently small $0<R_{0}=R_{0}(\Omega) \leq \sqrt{2} r_{0}$ and $0<R=R_{1}<$ $\min \left\{R_{0}, \sqrt{2} \rho, \sqrt{t_{1}}\right\}$ we can absorb the second term on the right of (4.9) in Lemma 4.2 on the left to obtain

$$
\sup _{0<r<R / \sqrt{2}} \beta_{t_{1}}(r) \leq C \sup _{x_{0} \in \Omega} \int_{0}^{R} B_{\left(x_{0}, t_{1}\right)}^{\varphi}(r) \frac{d r}{r} .
$$

But letting $r \downarrow 0$ in (4.4) and using (4.6), for any $x_{0} \in \Omega$, noting that (2.12) gives $H_{\left(x_{0}, t_{1}\right)}^{\varphi}(r) \rightarrow 0$ as $r \downarrow 0$, we find

$$
\int_{0}^{R} B_{\left(x_{0}, t_{1}\right)}^{\varphi}(r) \frac{d r}{r} \leq C H_{\left(x_{0}, t_{1}\right)}^{\varphi}(R)+C_{0} \delta(\rho, R),
$$

thus proving the claim.

Proof of Proposition 3.1 The claim follows from (4.4), (4.6), and Lemmas 4.1 and 4.3.

Proof of Proposition 3.2 For any $0<R<2 r<R_{1} \leq \min \left\{R_{0}, \sqrt{2} \rho, \sqrt{T / 2}\right\}$, any $x_{0} \in \bar{\Omega}$, and any $\left(x_{1}, t_{1}\right) \in Q_{r}\left(x_{0}, t_{0}\right)$, where $t_{0}=T-r^{2}$, we let $t_{2}=t_{1}+R^{2} / 4>t_{0}-r^{2}=T-2 r^{2}$ and choose $\left(x_{1}, t_{2}\right)$ as center of scaling. Then from (4.7), Lemma 4.1, and Lemma 4.3 letting $R_{2}^{2}=t_{2}-T+R_{1}^{2}>R_{1}^{2}-2 r^{2}>R_{1}^{2} / 2>R^{2} / 2$ we have

$$
\begin{aligned}
& R^{\mu-n-2} \int_{Q_{R / 2}\left(x_{1}, t_{2}-R^{2} / 4\right)}\left(|\nabla u|^{2}+|u|^{p}\right) d x d t \leq \frac{C}{R} \int_{R / 2}^{R / \sqrt{2}}\left(D^{\varphi}(r)+F^{\varphi}(r)\right) d r \\
& \leq C H_{\left(x_{1}, t_{2}\right)}^{\varphi}\left(R_{2}\right)+C R_{2} \sup _{x_{2} \in \Omega} H_{\left(x_{2}, t_{2}\right)}^{\varphi}\left(R_{2}\right)+C_{0} \delta\left(\rho, R_{2}\right) .
\end{aligned}
$$

But with (6.7) from [1], and observing that $t_{2}>T-2 r^{2}>2 r^{2}$ also gives $t_{2}>T / 2$, as in the proof of Proposition 6.2 in [1] for any $x \in \Omega$ we can bound $H_{\left(x, t_{2}\right)}^{\varphi}\left(R_{2}\right) \leq C H_{(x, T)}^{\varphi}\left(R_{1}\right)$ as well as $\delta\left(\rho, R_{2}\right) \leq C \delta\left(\rho, R_{1}\right)$, and the claim follows.

Proof of Proposition 3.4 Given $\rho>0$, by Proposition 3.1 and (2.6) with a constant $C_{0}=$ $C_{0}\left(E\left(u_{0}\right),\left\|u_{0}\right\|_{L^{2}}, T\right)>0$ for all $0<R \leq \min \left\{R_{0}, \sqrt{2} \rho, \sqrt{T / 2}\right\}$ there holds

$$
\sup _{x_{0} \in \Omega} H_{\left(x_{0}, T\right)}^{\varphi}(R) \leq C_{0} .
$$

We now may argue as in the proof of Proposition 6.8 in [1]. Fix $x_{0} \in \bar{\Omega}$ and suppose that for a sufficiently small number $\varepsilon>0$ to be determined and some number $0<R_{2} \leq R_{1} \leq$ $\min \left\{R_{0}, \sqrt{2} \rho, \sqrt{T / 2}\right\}$ there holds

$$
\sup _{\left|x_{1}-x_{0}\right|<2 R_{2} ; x_{1} \in \Omega} H_{\left(x_{1}, T\right)}^{\varphi}\left(R_{2}\right) \leq \varepsilon,
$$

where $R_{1}=R_{1}(\varepsilon)>0$ also is so small that our Proposition 3.1 together with (4.10) gives the bound

$$
H_{(x, T)}^{\varphi}(r) \leq H_{(x, T)}^{\varphi}(R)+\varepsilon / 2 \text { for all } x \in \bar{\Omega}, 0<r<R<R_{1},
$$


and so that Proposition 3.2 together with (4.10) and (4.11) yields the estimate

$$
\sup _{\left|x_{1}-x_{0}\right|<R_{2} ; x_{1} \in \Omega}\|u\|_{L^{p, \mu}\left(Q_{R / 2}\left(x_{1}, T-R^{2} / 4\right)\right)}^{p}<C \varepsilon
$$

for all $0<R<R_{2}$.

By the $\varepsilon$-regularity theorem, Proposition 4.1 in [1], for sufficiently small $\varepsilon>0$ we can then extend $u$ smoothly to the closure of $Q_{R_{2} / 10}\left(x_{0}, T\right)$. The details are exactly as in the proof of Proposition 6.8 in [1].

On the other hand, if (4.11) fails for every $0<R_{2}<R_{1}$, then as in the proof of Proposition 6.8 in [1] from (4.12) we find that $H_{\left(x_{0}, T\right)}^{\varphi}(R) \geq \varepsilon / 2=: \varepsilon_{0}$ for all $0<R<R_{1}$.

Proof of Proposition 3.5 By Rellich's compactness theorem, we may assume that $u_{k} \rightarrow u$ strongly in $L^{p-1}(\Omega \times] 0, T[)$, which suffices to show that $u$ solves (1.1) on $\Omega \times[0, T$ [ in the sense of distributions. Moreover, boundedness of $\left(u_{k}\right)$ in $H^{1} \cap L^{p}(\Omega \times[0, T[)$ ensures that for every $\tau>0$ there exists a constant $C=C(\tau)$ with

$$
\sup _{2 \tau \leq t_{0}<T} \sup _{x_{0} \in \Omega} H_{u_{k},\left(x_{0}, t_{0}\right)}^{\varphi}(R) \leq C, \text { uniformly in } 0<R<\sqrt{\tau} .
$$

The remainder of the proof is similar to the proof of partial regularity of the tangent map $\bar{u}$ in [1], Theorem 6.9. Indeed, given any compact set $Q \subset \bar{\Omega} \times] 0, T]$ with a suitable number $\varepsilon>0$ we have $\operatorname{sing}(u) \cap Q \subset S$, where

$$
S=\left\{z_{0}=\left(x_{0}, t_{0}\right) \in Q ; \liminf _{r \downarrow 0} r^{\mu-n-2} \liminf _{k \rightarrow \infty} \int_{P_{r}\left(z_{0}\right)}\left(\left|\nabla u_{k}\right|^{2}+\left|u_{k}\right|^{p}\right) d z>\varepsilon\right\},
$$

and a standard covering argument yields that $\mathcal{H}^{n+2-\mu}(S)<\infty$.

Proof of Theorem 3.7 The only necessary modification concerns the bound for the error term $I_{0}^{\varphi}$ in the proof of Theorem 6.9 in [1], which now reads

$$
I_{0}^{\varphi}=\frac{2}{p} \int_{0}^{R_{0}}\left(\left|A_{0}^{\varphi}(r)\right|+\left|B_{-}^{\varphi}(r)\right|\right) \frac{d r}{r} \leq C_{0} \delta\left(\rho, R_{0}\right)+C R_{0} \rightarrow 0 \quad\left(R_{0} \downarrow 0\right)
$$

on account of Proposition 2.1 and Lemmas 4.1 and 4.3.

Proof of Theorem 3.8 Recall that by Proposition 3.4 for any given $\rho>0$ we have $\operatorname{sing}(u) \cap$ $\Omega \times\{T\} \subset S^{T}$, where

$$
S^{T}=\left\{z_{0}=\left(x_{0}, T\right) \in \bar{\Omega} \times\{T\} ; H_{\left(x_{0}, T\right)}^{\varphi}(R) \geq \varepsilon_{0} \quad \text { for all } 0<R<R_{1}\right\},
$$

with $\varphi=\varphi^{\rho}$ and with $R_{1}, \varepsilon_{0}>0$ as defined in Proposition 3.4. But via an estimate similar to (6.3) in [1] and Young's inequality, letting $D=D_{\left(x_{0}, T\right)}^{\varphi}(R)$, etc., with a constant $C_{1}=C_{1}\left(\varepsilon_{0}\right)>0$ we can bound

$$
H_{\left(x_{0}, T\right)}^{\varphi}(R) \leq \frac{2}{p}\left(D-F_{p}+\frac{1}{p-2} F_{p}^{2 / p}\right) \leq C_{1}\left(D+F_{p}\right)+\varepsilon_{0} / 2
$$

for all $x_{0} \in \bar{\Omega}$ and all $0<R<R_{1}$. For each $x_{0} \in S^{T}$ and any $0<R<R_{1}$ we then find that

$$
\varepsilon_{0} / 2 \leq C_{1}\left(D(R)+F_{p}(R)\right) \leq C_{1} R^{\mu} \int_{\Omega \times\left\{T-R^{2}\right\}}\left(|\nabla u|^{2}+|u|^{p}\right) G_{\left(x_{0}, T\right)} d x .
$$


Following the argument of [2], given $0<R<R_{1}$ by Vitali's theorem we can choose a collection $\left(B_{R}\left(x_{j}\right)\right)_{1 \leq j \leq J}$ of balls with centers $x_{j} \in S^{T}$ for each $j$ such that $S^{T} \subset$ $\cup_{1 \leq j \leq J} B_{R}\left(x_{j}\right)$ and with

$$
\left|x_{j}-x_{k}\right| \geq R \text { for } 1 \leq j<k \leq J .
$$

Setting $z_{j}=\left(x_{j}, T\right)$ for each $j$, by [2], Lemma 1, with a uniform constant $C>0$ independent of $R$ there holds

$$
\sum_{1 \leq j \leq J} G_{z_{j}}(x, t) \leq C R^{-n} \text { uniformly in }(x, t) \in \Omega \times\left[T-4 R^{2}, T-R^{2}[.\right.
$$

From (4.16) for $0<R<R_{1} / 2$ we then find that

$$
\begin{aligned}
J R^{n+2-\kappa-\mu} \varepsilon_{0} & \leq 2 C_{1} R^{n-\kappa} \sum_{1 \leq j \leq J} \int_{T-4 R^{2}}^{T-R^{2}} \int_{\Omega}\left(|\nabla u|^{2}+|u|^{p}\right) G_{z_{j}} d x d t \\
& \leq C R^{-\kappa} \int_{T-4 R^{2}}^{T} \int_{\Omega}\left(|\nabla u|^{2}+|u|^{p}\right) d x d t .
\end{aligned}
$$

In view of (3.6) the right hand side in the previous expression is uniformly bounded for a sequence of numbers $R=R_{k} \downarrow 0$, and the claim follows.

\section{Proof of Theorem 3.10}

\subsection{Boundary monotonicity}

Suppose that $\Omega$ is convex and let $0 \leq u_{0} \in H_{0}^{1} \cap C^{1}(\bar{\Omega})$. By standard parabolic theory there exists $t_{0}>0$ such that the initial value problem for (1.1) with initial data $\left.u\right|_{t=0}=u_{0}$ admits a smooth solution $u$ on $\Omega \times\left[0, t_{0}\right]$, which may be extended to a classical solution on a maximal time interval $\left[0, t_{\max }\right.$ [ for some $t_{\max } \leq \infty$. Clearly we may assume that $u_{0} \neq 0$; hence $u(t)>0$ for $t>0$ by the strong maximum principle. Moreover, by the Hopf boundary maximum principle there is $\delta>0$ such that for every $x_{0} \in \partial \Omega$ at the time $t_{0}$ there holds

$$
v\left(x_{0}\right) \cdot \nabla u\left(x, t_{0}\right)<0 \text { for all } x \in \Omega \cap B_{\delta}\left(x_{0}\right) .
$$

Following a suggestion of DeMarchis-Malchiodi-Martinazzi [4], we now use the Alexandrov reflection principle (also called the technique of moving planes), as pioneered by Gidas-NiNirenberg [6], to show monotonicity of $u$ near the boundary for all $t \geq t_{0}$.

Given $x_{0} \in \partial \Omega$ we may translate the origin and also rotate the axes of our coordinate system to achieve that $x_{0}=0$ with exterior normal $v\left(x_{0}\right)=(-1,0, \ldots, 0)$. Writing $x=\left(x_{1}, x^{\prime}\right)$ for $x \in \mathbb{R}^{n}$, for $\tau>0$ let

$$
T_{\tau}:=\left\{x \in \bar{\Omega} ; x_{1}=\tau\right\}
$$

and denote as

$$
x^{\tau}:=\left(2 \tau-x_{1}, x^{\prime}\right)
$$

the image of the point $x=\left(x_{1}, x^{\prime}\right)$ after reflection in $T_{\tau}$. Also set

$$
\Sigma(\tau):=\left\{x \in \Omega ; x_{1}<\tau\right\}, \Sigma^{\prime}(\tau):=\left\{x^{\tau} ; x \in \Sigma(\tau)\right\},
$$


and let

$$
\tau_{0}:=\sup \left\{\tau ; \quad \Sigma^{\prime}(\tau) \subset \Omega \quad \text { and } \quad \nu_{1}(x)<0 \quad \text { for } x \in \partial \Sigma(\tau) \backslash T_{\tau} \subset \partial \Omega\right\} .
$$

Note that $\tau_{0}>0$ in view of smoothness of $\partial \Omega$. Finally, for $0<\tau<\tau_{0}$ let

$$
\tilde{u}(x, t):=u\left(x^{\tau}, t\right), x \in \Sigma(\tau) .
$$

Observe that $\tilde{u}$ again is a solution to the equation (1.1). Also note that since $u(t)>0$ in $\Omega$ for any $t>0$, we have

$$
u \not \equiv \tilde{u} \text { on } \Sigma(\tau) \times\left[t_{0}, t_{1}\right]
$$

for all $0<\tau<\tau_{0}$ and all $t_{1} \in\left[t_{0}, t_{\max }\right.$ [. Replacing $\tau_{0}>0$ with a smaller number, if necessary, by (5.1) we finally may assume that at time $t=t_{0}$ we have

$$
\inf _{x \in \Sigma(\tau)} u_{x_{1}}(x, t)>0, u(x, t)<\tilde{u}(x, t) \text { for all } x \in \Sigma(\tau), 0<\tau \leq \tau_{0} .
$$

Proposition 5.1 The strict inequalities (5.3) hold for every $t \in\left[t_{0}, t_{\max }[\right.$.

Proof Since (5.3) holds at time $t=t_{0}$ there is $t_{1}>t_{0}$ such that (5.3) holds for $t_{0} \leq t \leq t_{1}$. Assume by contradiction that (5.3) fails to hold for some minimal $\left.t_{2} \in\right] t_{0}, t_{\max }[$.

Setting $w=\tilde{u}-u$ we have that $w \geq 0, w \not \equiv 0$ on $\Sigma\left(\tau_{0}\right) \times\left[t_{0}, t_{2}\right]$ with $w=0$ on $T\left(\tau_{0}\right) \times\left[t_{0}, t_{2}\right]$. Moreover, in view of (1.1) there holds

$$
w_{t}-\Delta w=c w \text { on } \Sigma\left(\tau_{0}\right) \times\left[t_{0}, t_{2}\right],
$$

where

$$
c(x, t)=(p-1) \int_{0}^{1}|(1-s) u(x, t)+s \tilde{u}(x, t)|^{p-2} d s
$$

is smooth and bounded. From the strong maximum principle then it follows that $w>0$, that is,

$$
u(x, t)<\tilde{u}(x, t) \text { for all }(x, t) \in \Sigma\left(\tau_{0}\right) \times\left[t_{0}, t_{2}\right] ;
$$

in addition, thereby using the boundary maximum principle for the equation (5.4) at points $x \in \Omega \cap T\left(\tau_{0}\right)$ and for the original flow equation (1.1) at points $x \in \partial \Omega \cap \Sigma\left(\tau_{0}\right)$, together with the fact that $v_{1}(x)<0$ for any such point, we find that

$$
u_{x_{1}}(x, t)>0, \quad \text { for all }(x, t) \in \partial \Sigma\left(\tau_{0}\right) \times\left[t_{0}, t_{2}\right] .
$$

But $\tilde{w}:=u_{x_{1}} \geq 0$ also is a solution of (5.4) with $\tilde{c}=(p-1)|u|^{p-2}$ in place of $c$. Since $u_{x_{1}}>0$ on $\partial \Sigma\left(\tau_{0}\right) \times\left[t_{0}, t_{2}\right]$, from the maximum principle it follows that (5.3) also holds for all $x \in \Sigma\left(\tau_{0}\right)$ at time $t=t_{2}$, contrary to our assumption about $t_{2}$.

By compactness of $\partial \Omega$ there exists a number $\delta_{0}>0$ such that $\tau_{0} \geq \delta_{0}$ for all $x_{0} \in \partial \Omega$, and by Proposition 5.1 for every $x_{0} \in \partial \Omega$ and for every $t \in\left[t_{0}, t_{\max }\right.$ [ there holds

$$
v\left(x_{0}\right) \cdot \nabla u\left(x_{0}-s v\left(x_{0}\right), t\right)<0 \text { for all } 0 \leq s \leq \delta_{0},
$$

as we see from (5.3) after shifting $x_{0}=0$ and rotating coordinates suitably as before; that is, $u(t)$ is monotonically decreasing in outward normal direction in a uniform neighborhood of $\partial \Omega$ for every $t \in\left[t_{0}, t_{\max }\right.$ [. Clearly we may assume in addition that $\delta_{0}$ is less than half the focal distance from $\partial \Omega$.

As an immediate corollary to Proposition 5.1 we obtain the following result. 
Proposition 5.2 Let $\delta_{0}>0$ be as in (5.5) and suppose that $x_{0} \in \partial \Omega$ with $\left(x_{0}, T\right) \in \operatorname{sing}(u)$. Then for every $0 \leq s \leq \delta_{0}$ we have $\left(x_{0}-s v\left(x_{0}\right), T\right) \in \operatorname{sing}(u)$.

Proof If we suppose that $z_{s}:=\left(x_{0}-s v\left(x_{0}\right), T\right) \notin \operatorname{sing}(u)$ for some $0 \leq s \leq \delta_{0}$, by definition of $\operatorname{sing}(u)$ there exist $r>0, M>0$ such that $|u|<M$ in $Q_{r}\left(z_{s}\right)$. By (5.5) then with a constant $\gamma=\gamma(\Omega)>0$ depending only on a bound for the principal curvatures of $\partial \Omega$ we have $|u|<M$ in $Q_{\gamma r}\left(x_{0}, T\right)$, and $\left(x_{0}, T\right) \notin \sin g(u)$ by parabolic regularity.

\subsection{Proof of Theorem 3.10}

Letting

$$
S_{T}:=\operatorname{sing}(u) \cap \Omega \times\{T\}, \partial S_{T}:=\operatorname{sing}(u) \cap \partial \Omega \times\{T\},
$$

by Proposition 5.2 we have

$$
X_{T}:=\left\{\left(x_{0}-s v\left(x_{0}\right), T\right) ;\left(x_{0}, T\right) \in \partial S_{T}, 0 \leq s \leq \delta_{0}\right\} \subset S_{T} .
$$

But for our choice of $\delta_{0}$ the set $X_{T}$ is homeomorphic to $\partial S_{T} \times\left[0, \delta_{0}\right]$ via a uniformly bi-Lipschitz map. Thus, by Theorem 3.6 of [5] for any $0<\alpha \leq n-1$ we have

$$
\delta_{0} \mathcal{H}^{\alpha}\left(\partial S_{T}\right) \leq C \mathcal{H}^{\alpha+1}\left(X_{T}\right) \leq C \mathcal{H}^{\alpha+1}\left(S_{T}\right) .
$$

(We are indebted to Andrea Mondino for providing reference [5].) Theorem 3.10 thus follows from Theorem 3.8. The proof is complete.

The references given below by far do not exhaust the topic. See our paper [1] for a more extensive list of results on the Lane-Emden equation and its background.

\section{References}

1. Blatt, S., Struwe, M.: An analytic framework for the supercritical Lane-Emden equation and its gradient flow Int. Math. Res. Notices (2014). doi:10.1093/imrn/rnt359

2. Cheng, X.: Estimate of the singular set of the evolution problem for harmonic maps. J. Differ. Geom. 34(1), 169-174 (1991)

3. Chou, K.-S., Du, S.-Z., Zheng, G.-F.: On partial regularity of the borderline solution of semilinear parabolic problems. Calc. Var. Partial Differ. Equ. 30(2), 251-275 (2007)

4. De Marchis, F., Malchiodi, A., Martinazzi, L.: Private communication (2013)

5. Ernst, L.R., Freilich, G.: A Hausdorff measure inequality. Trans. Am. Math. Soc. 219, 361-368 (1976)

6. Gidas, B., Ni, W.M., Nirenberg, L.: Symmetry and related properties via the maximum principle. Commun. Math. Phys. 68(3), 209-243 (1979)

7. Giga, Y., Kohn, R.V.: Asymptotically self-similar blow-up of semilinear heat equations. Commun. Pure Appl. Math. 38(3), 297-319 (1985)

8. Holland, C.J.: Limiting behavior of a class of nonlinear reaction diffusion equations. Q. Appl. Math. 40(3), 293-296 (1982/83)

9. Matano, H., Merle, F.: On nonexistence of type II blowup for a supercritical nonlinear heat equation. Commun. Pure Appl. Math. 57(11), 1494-1541 (2004)

10. Matano, H., Merle, F.: Threshold and generic type I behaviors for a supercritical nonlinear heat equation. J. Funct. Anal. 261(3), 716-748 (2011)

11. Ni, W.-M., Sacks, P.E., Tavantzis, J.: On the asymptotic behavior of solutions of certain quasilinear parabolic equations. J. Differ. Equ. 54(1), 97-120 (1984)

12. Pacard, F.: Partial regularity for weak solutions of a nonlinear elliptic equation. Manuscr. Math. 79(2), 161-172 (1993)

13. Struwe, M.: On the evolution of harmonic maps in higher dimensions. J. Differ. Geom. 28(3), 485-502 (1988)

14. Weissler, F.B.: Existence and non-existence of global solutions for a semilinear heat equation. Isr. J. Math. 38(1-2), 29-40 (1981) 